\title{
SUL SIGNIFICATO DELLA SCULTURA AFRICANA ${ }^{1}$
}

\section{VINIGI L. GROTTANELLI}

$\mathrm{I}^{\mathrm{A}}$ A conoscenza della scultura africana ha avuto una storia paradossale. Nei secoli in cui fioriva in Europa la moda dell'esotismo orientaleggiante, quest'arte esotica per eccellenza fu totalmente ignorata; ma essa divenne celebre nel momento in cui l'esotismo tramontava. I suoi capolavori più genuini furono raccolti a migliaia quando quasi nessuno sospettava il loro intrinseco valore; acquistarono iperbolica fama ad opera di persone che ignoravano tutto a loro riguardo, e furono esaltate proprio per quei pregi che in realtà non possedevano. Nello stesso momento in cui la plastica negra rappresentava un lievito di grande importanza, per quanto male impiegato, nella cultura occidentale, gli Europei ne causavano il completo tracollo. $\mathrm{E}$ infine, fenomeno ancora più malinconico, gli Africani acquistano consapevolezza riflessa della loro nobilissima tradizione artistica proprio oggi, in un mondo che, a meno di miracoli, sembra aver posto su quella tradizione una triste pietra tombale.

Questo autentico groviglio di apparenti contraddizioni ha le sue cause e $\mathrm{i}$ suoi riflessi in campi - culturali, sociali, politici, umani in senso lato - che hanno con l'arte rapporto solo indiretto. Di essi ogni persona colta ha oggi conoscenza, e ciò mi consente di limitarmi stasera a considerare gli aspetti puramente storico-artistici del problema, forse meno noti.

La fortuna della scultura africana in Europa si inizia nell'anno 1904. Tutti conoscono l'aneddoto che ne consacra l'inizio, quando Maurice de Vlaminck s'incapriccia di certe figurine - del Dahomey, si dice - scovate fra le bottiglie di 'fine' e di Pernod dietro il banco di un vinaio di Argenteuil, le acquista per pochi franchi, per cederne poi una a Derain e scatenare d'improvviso i lirici entusiasmi di Apollinaire e più tardi di Pablo Picasso.

Ho detto di proposito 'fortuna' della scultura africana, e non 'scoperta', perché la vera scoperta di quest'arte risale a epoca molto più lontana. Già alla fine del Quattrocento, navigatori portoghesi avevano rivelato all'occidente le sorprendenti plastiche - bronzi, legni e avori - dell'allora raggiunta costa di Guinea. Quella insospettata perizia scultorea degli artefici negri ne stimolò ad opera degli occidentali una produzione 'su commissione', che trovò favore alle corti europee : forse alcuni artisti guineani furono attivi anche in Portogallo. Si tratta comunque di uno dei primi fenomeni noti di parziale acculturazione euro-africana, le cui più celebri testimonianze sono date dai cucchiai, corni da caccia e coppe d'avorio detti ' di Benin' anche se provenienti da altri centri della Guinea; alcune di queste opere sono ancora conservate nei nostri musei. ${ }^{2}$ Apprezzate come esemplari di curiosità, esse non suscitarono, che si sappia, nessun impulso fra gli artisti europei dell'epoca, volti a ben altri ideali e modelli; allo stesso modo come l'ammirazione di un Dürer o di

The 196I Lugard Memorial Lecture, delivered by Professor Grottanelli in Rome on I4 April 1961, on the occasion of the annual meeting of the Executive Council of the Institute.

${ }^{2}$ R. Pettazzoni, Avori scolpiti africani in collezioni italiane, Roma, I912; W. B. Fagg, Afro-Portuguese Ivories, London, s.d.; e per la bibliografia, S. Wolf, 'Afrikanische Elfenbeinlöffel des 16 . Jahrhunderts im Museum für Völkerkunde Dresden', in Ethnologica, N.F., Bd. 2, Köln, 1960, pp. 410-25. 
un Cellini di fronte ai rari cimeli messicani importati alle corti imperiali o medicee ha lasciato traccia solo nelle annotazioni dei loro biografi.

Per oltre tre secoli, l'Africa non fornisce all'arte occidentale che secondari spunti di colore e di costume, i quali per di più sono moreschi più che africani. Ancora nella sua massima glorificazione programmatica, nei grandi affreschi tiepoleschi dello scalone di Würzburg, nei quali l'Asia sciorina simbolicamente i tesori delle sue arti, l'allegoria moresca del continente nero non ha da esibire che sgargianti costumi e più o meno improbabili animali.

Il XIX secolo, specie nella seconda metà, e i primi due o tre decenni del XX, vedono la raccolta - dapprima occasionale e poi via via più sistematica - di ingenti collezioni etnografiche da ogni parte del continente, fra le quali sono casualmente inclusi quelli che più tardi verranno riconosciuti come 'oggetti d'arte'. Parallelamente, la penetrazione europea comincia a rompere l'equilibrio tradizionale delle società africane, soppianta le antiche tecniche introducendone di nuove, fa vacillare o crollare i sistemi di credenze che inspiravano gli artefici locali. Tra la fine dell'Ottocento e i primi del Novecento, si compiono dunque due processi concomitanti che rimarranno fondamentali per tutti gli studi successivi sull'argomento. $\mathrm{Da}$ un lato, una parte ingente della produzione artistica africana, e forse la migliore, si trasferisce dai luoghi di origine alle collezioni e musei d'Europa e d'America. Dall'altro lato, s'inaridiscono e scompaiono quasi ovunque i centri creativi di quelle arti, o si degradano a centri di mediocre artigianato turistico-commerciale.

Al punto in cui siamo oggi, nel $196 \mathrm{r}$, l'inventario tipologico della scultura africana è pressoché completo e definitivo. La speranza di ritrovare ancora un qualche centro. incontaminato di produzione artistica deve essere abbandonata: il continente non ha più popoli o 'angoli' inesplorati, non rimane praticamente più nulla da 'scoprire '. Quest'arte si studia oggi sui manuali, sulle fotografie dei libri, o nelle vetrine dei musei non diversamente da quella delle civiltà estinte e sepolte; e, tolte rare eccezioni a cui si accennerà, tale studio ha il carattere anatomico di un'autopsia eseguita su tavolo operatorio, più che di un'indagine fisiologica su un organismo vivente.

Ecco dunque i presupposti di fatto da cui hanno preso le mosse le diverse indagini e valutazioni sulla plastica negra. A questo punto va fatta un'osservazione preliminare che è importantissima, benché - o forse appunto perché - la si possa trovare del tutto naturale. $E$ cioè questa, che praticamente tutte le valutazioni date finora di queste forme d'arte sono state date da Europei (o Americani) senza tenere in minimo conto i criteri e giudizi degli Africani stessi. Le ragioni di questo modo di procedere possono essere tre. O non si conoscevano affatto le reazioni degli Africani competenti nel caso singolo (come per gli oggetti d'incerta provenienza o di vecchia data di raccolta); o si riteneva che il loro pensiero al riguardo fosse irrilevante in linea di principio; oppure si pensava che il loro apprezzamento, anche se conosciuto, avrebbe necessariamente coinciso con quello dato dagli Europei, in omaggio a una presunta universalità dei valori estetici (idea strana, in quanto proprio le profonde differenze fra arte negra e arte europea parevano di per sé lasciar supporre tutto il contrario).

Comunque, le reazioni dei Bianchi di fronte all'arte africana devono essere divise in due categorie abbastanza nette: quelle degli esteti, letterati e artisti da un lato, quelle degli etnologi dall'altro. Le reazioni del c.d. pubblico medio rientrano in una 
terza categoria, non priva d'interesse, ma che può essere trascurata qui : non perché riflettente opinioni meno significative, ché anzi essa era ed è probabilmente più genuina e spregiudicata delle altre, ma perché più incerta e fluttuante e difficilmente controllabile in quanto anonima e raramente tradotta in giudizi scritti, quando non influenzata dalle valutazioni delle altre due categorie dette. Occupiamoci dunque di queste.

\section{L'EQUIVOCO DEGLI ESTETI}

Il programma ideologico del Fauvisme, sorto in violenta reazione al convenzionalismo della ' civiltà '-e con il quale nasce ai primi del nostro secolo la ' moda' africana - portava implicita in sé una rivalutazione dei c.d. 'primitivi', a cui si attribuivano genericamente, a torto $\mathrm{o}$ a ragione, spontaneità di espressione, impeto e audacia nell'impiego di colori violenti e di forme ardite. Allo stesso modo in cui un personaggio di Gide in quegli anni confessava di 'cercare nei libri la giustificazione delle sue opere', i Fauves credettero trovare nella scultura negra una giustificazione ante litteram delle nuove tendenze e del loro credo estetico.

La loro indifferenza al significato originario di quella scultura è pari a quella già mostrata da Gauguin nei riguardi dell'arte polinesiana. L'esaltazione di quelle forme è, per i Fauves, di natura letteraria e intellettuale, e si accompagna all'atteggiamento di rivolta contro i canoni prospettici della spazialità, incontrastatamente rispettati dal Rinascimento in poi.

Affermare che al difuori di tali canoni si possano ideare veri capolavori era idea tanto più audace in quanto fino allora nessuno fra gli artisti né fra il pubblico, né fra gli stessi etnologi, aveva riconosciuto una così alta qualifica alle 'assurde' produzioni plastiche negre.

Grazie a un'interpretazione non molto diversa, cioè in quanto considerate documenti ingenui di un sentimento primordiale, quelle sculture divennero popolari anche fra gli espressionisti tedeschi del gruppo Die Brücke e del Blaue Reiter: pare che Ernst Kirchner 'scoprisse' la scultura africana nel Museo di Dresda allo stesso tempo di Vlaminck, e indipendentemente da lui. Kandinskij e il suo gruppo cercano le limpide sorgenti dell'ispirazione nella purezza originaria dei popoli selvaggi non meno che nell'ingenuità folkloristica del volgo europeo; e Franz Marc prova un'intima gioia 'al pensiero che già una volta vi erano uomini primordiali che amavano l'astratto come noi '; e polemizzando contro lo spirito sentimentale dell'eredità romantica aggiunge: ' L'uomo primitivo non aveva sentimentalismi, quando amava l'astratto.'I

E abbastanza chiaro che esteti e artisti, agli inizi del secolo e per parecchio tempo ancora, ammettevano ' $n$ 'éprouver vis-à-vis des idoles nègres que des sensations esthétiques et d'évocation poétique', come dice Apollinaire, ma al tempo stesso s'interessavano sia pure ingenuamente al problema 'de fixer l'époque certaine des plus beaux de ces fétiches de bois, dont certains remontent à une très haute antiquité ' $;^{2}$ e in fondo non sapevano sottrarsi alla tentazione di fantasticare a modo

I U. Apollonio, 'Espressionismo', in Enciclopedia Universale dell'Arte, v, 66, Venezia-Roma (1961); A. Rossi, 'Il primitivo nella scultura contemporanea', in Il Contemporaneo, ii. I 7 (Settembre 1959); H. Sedlmayr, 'Franz Marc oder die Unschuld der Tiere', in Wort und Wahrheit, giugno $195 \mathrm{x}$; lo stesso, La rivoluzione dell' arte moderna, Milano, 1960 , pp. I I I sgg.

${ }^{2} \mathrm{Cit}$. in Introduction, Arts d'Afrique et d'Océanie, Cannes, I957. 
loro sul significato arcano di quei modelli apparentemente incomprensibili, di collocarli in un supposto mondo di emozioni 'originarie ', spostando impercettibilmente il loro interesse dalla forma al contenuto.

I comodi clichés, gli apprezzamenti superficiali sugli Africani, che potevano giustificare i più fantasiosi giudizi sui moventi della loro arte, non erano del resto solo frutto dell'immaginazione o dell'ignoranza di pittori e scultori ai primi del secolo. Questi potevano attingere copiosamente ai testi di etnologi esordienti e anche qualificati dell'epoca. Vien fatto di pensare alle comiche astrazioni che facevano ridere il compianto Marcel Griaule: 'Si possono riconoscere fra i Sudanesi la glorificazione dello stato, legami di parentela improntati a tenerezza, una viva religiosità, e l'uso della zappa in agricoltura '. I'ingenuo cliché del Negro 'dipinto come flemmatico per il suo umore pacato, e come feticista per le innumerevoli superstizioni che ne annebbiano la mente' si ritrova ancora nel 1943 nelle pagine, non meno ricche di involontaria vis comica, di un etnografo partenopeo. Leo Frobenius ha scritto che 'l'idea di un Negro barbaro e feticista è un'invenzione europea che ha dominato l'Europa fino all'inizio di questo secolo '; 2 ma si sbagliava nel termine ad quem.

Ma anche fuori dell'umorismo, scrittori insigni come L. Lévy-Bruhl sostenevano ancora fra il 1923 e il 1927 che il 'primitivo' non comprende nessuna cosa come noi, spiega ogni fatto con cause mistiche, e non sa far nulla senza mezzi magici; il che, se vero, doveva scoraggiare chiunque dall'intraprendere uno studio storicoartistico della plastica africana. Nel 1928 , al suo ritorno da un viaggio nelle zone artisticamente più feconde dell'Africa, il più acuto e sensibile dei letterati francesi scriveva: 'Les gens de ces peuplades primitives, je m'en persuade de plus en plus, n'ont pas notre façon de raisonner; c'est pourquoi ils nous paraissent bêtes . . . Les rapports de causalité semblent n'exister point pour eux.'3

E un africanista fra i più quotati scriveva dei Negri africani nel $1925: ' \ldots$ il est incontestable que leur mentalité globale se rapproche, par beaucoup de points, de la mentalité primitive ... Le mysticisme et le collectivisme sont à la base de toutes leurs manifestations d'ensemble .. .'4 Nessun critico sarebbe stato preso sul serio se avesse cercato di spiegare con banalità così generiche - fede primitiva, misticismo, collettivismo - la genesi, p.es., della scultura gotica: eppure le stesse grossolane spiegazioni furono accettate come vere e profonde per la scultura negra. E legittimo allora porsi la domanda, se da un'incomprensione totale della natura dell'arte africana potesse discendere una fruttuosa adozione delle sue forme e motivi in sede figurativa. Non spetta all'etnologo in quanto tale esprimere un parere in campo storico-artistico, ma egli sarà tuttavia autorizzato ad affermare questo : che in tutti i casi in cui i critici credono scorgere suggestioni, reminiscenze, $o$ altre trasposizioni di autentici elementi africani nelle opere (pittoriche o scultoree) dei loro ammiratori occidentali, la loro contaminazione e deformazione è di tale natura da apparire agli occhi di un etnologo poco meglio o poco peggio di una puerile parodia.

Questa impressione rimane inalterata comunque si voglia o possa interpretare

'M. Griaule, Arts de l'Afrique Noire, Paris, 1947, p. 9.

${ }^{2} \mathrm{~L}$. Frobenius, Histoire de la civilisation africaine, Paris, 1933, p. Is.
3 A. Gide, Le Retour du Tchad, Paris, I928, Pp. 4849.

4 M. Delafosse, Civilisations négro-africaines, Paris, I 925 , p. 8. 
l'intenzione che sta alla base di queste dichiarate o supposte analogie. Intenzione che può essere in certi casi quella di una trasposizione deliberata, come nel grande bronzo di Jacques Lipschitz al Museum of Modern Art di New York, in cui le braccia ' ad ansa di marmitta' rammentavano a Léon Kochnitzky quelle dei feticci chiodati del Mayombe, mentre la testa ricorda una maschera funeraria dei BaKota; o può essere un'allusione satirica, come nel celebre 'Schamloses Tier' del giovane Paul Klee, ispirata o copiata da uno dei c.d. 'feticci terapeutici' dei Bushongo; o derivare dalla distorsione di un soggetto Dan o Rega, come in una maschera del primo Picasso; ' e cosi via. Occorre anche dire che gli ' africanismi' di alcune personalità artistiche più significative sono più che altro apparenti, suggeriti dall'aura creata intorno ad esse dai critici. Prendiamo il caso di Matisse. Secondo la nota testimonianza di Apollinaire nel suo 'Médaillon', questo 'astuto fauve ... amava circondarsi di quelle sculture in cui i Negri della Guinea, del Senegal e del Gabun hanno dimostrato con purezza unica le loro spaventate emozioni ', e Berenson ce lo conferma dopo la sua visita del 1950. Ma individuare concretamente imprestiti o sia pure suggestioni dell'arte negra nelle tele di Matisse, o nelle sue stesse opere scultoree (come nel Torso avec une tête o ne La Serpentine, p.es., o anche nelle Deux Négresses) è altra cosa: sarà più propenso a farlo il critico d'arte che non l'etnologo esperto di scultura africana.

Comunque, è bene lasciare ai critici il problematico processo alle intenzioni, e (quando sia il caso) la ricerca degli eufemismi che mascherino la parola da non impiegarsi, 'imitazione'. Rimane per noi il fatto che quelle derivazioni, chiamiamole così, più se ne vedono (e ce n'è di tutti i colori) più tradiscono l'incapacità di mantenere proprio $i$ valori delle opere originali che più si proclamano, cioè il loro candore, il loro misticismo, la loro supposta primitiva naturalezza.

Ciò è stato riconosciuto, sia pur prudentemente, da qualche critico in vena di sincerità: 'L'artista di oggi si illude di ripetere atteggiamenti primitivi in modo primitivo, ma questa ripetizione non è che una manifestazione culturale di gusto decisamente moderno, che in grandi artisti come Picasso e Moore raggiunge sì la sincerità, ma quella sincerità che è . . . risolutrice di problemi solamente moderni. Spesso accade di peggio: queste immagini arcaiche e mitiche divengono per molti artisti (ed è la maggioranza) solo motivo di evasione, un fatto di intellettualismo fine a se stesso... Il primitivismo, sia quello positivo di Picasso, sia quello negativo al quale ora si è accennato, rimane ovviamente, mediato e riflesso com'è, sul piano della sola intenzione; le Teste di Modigliani o le Donne in bronzo di Picasso sono primitive come sono primitivi i Ritmi della primavera di Stravinsky, cioè non lo sono affatto.'2

All'etnologo pensoso di tali problemi della nostra cultura, l'equivoco (per non dire il fallimento) in questione appare motivato da due cause fondamentali. La prima è l'estrema problematicità, per non dire l'impossibilità, di raggiungere risultati positivi e costruttivi di acculturazione quando a quest'ultima manchi una base di comprensione culturale reciproca, presupposto di ogni processo di integrazione. Questa è una verità etnologica di ordine generale, non infirmabile nel campo della storia dell'arte e in nessun altro campo della cultura. (In base alla medesima verità si potrebbe

\footnotetext{
I L. Kochnitzky, Influence de la plastique nègre sur l'art contemporain, éd. 'Synthèses', Bruxelles, s.d., 
anche dire che per parte loro gli Africani non avrebbero approdato a risultati migliori se si fossero messi a scolpire o dipingere lasciandosi influenzare, che so io, dai primitivi Senesi o dai Preraffaelliti. Essi meritano se non altro il nostro rispetto per non averlo tentato.)

La seconda causa dell'equivoco, più specifica ma derivante in ultima analisi dalla prima, non è imputabile a mancanza di sincerità o a superficialità degli artisti occidentali, ma piuttosto a un grosso malinteso corrente nel mondo in cui essi vivevano e vivono, dal quale solo gli etnologi - e solo in anni recenti, e non tutti - si sono liberati. E cioè la verità è questa, anche se non posso qui dimonstrarvela perché richiederebbe un lungo discorso: la scultura africana, come l'arte detta primitiva in genere, non è essa stessa ' primitiva' affatto, come non sono per nulla primitivi gli uomini che la creano. Non siamo quasi mai di fronte ad abbozzi primordiali, a espressioni spontanee di un supposto (e inesistente) Naturmensch, bensì al contrario, ai prodotti di secolari e molto elaborate tradizioni. Non opere di esuberante gioventù o tanto meno di inesperta fanciullezza : ma opere di consapevole e pensosa maturità, per non dire di esperienza senile. Non punti di partenza, ma punti d'arrivo. Tutte le fantasticherie costruite sulla falsa impalcatura di questo ' primitivismo', con i loro corollari psicologici ed estetici, crollano come un castello di carte. Si è a lungo creduto di inspirarsi a un 'primitivo' che non esiste.

Poiché si è parlato dei rapporti fra scultura africana e artisti contemporanei, si può aggiungere una parola circa il merito che questi ultimi avrebbero avuto attirando l'attenzione del pubblico sulla esistenza, prima d'allora ignorata o addirittura negata, di una vera e propria arte dei Negri africani:

Questo merito è stato contestato, o almeno posto in discussione, da autori recenti,

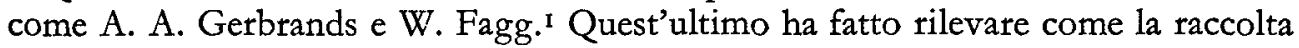
di queste e altre opere esotiche - compiuta con competenza e discernimento - sia benemerenza di etnologi, viaggiatori e conservatori di musei, almeno fin dai primi del secolo scorso; che senza l'intelligente opera di essi, gli esteti non avrebbero avuto nulla da scoprire; anzi, che quei medesimi oggetti erano rimasti per decenni bene esposti nelle vetrine senza che nessuno di quegli esteti si fosse mai data la pena di degnarli di uno sguardo.

Do atto di leale spirito di corpo ai miei colleghi etnologi; ma per obiettività mi pare che in questa piccola controversia si debba segnare un punto a favore degli artisti. Perché in verità a quale giuria può addebitarsi quella accusa di indifferenza e cecità? Se la cronaca è esatta nel datare al 1904 l'esplosione di entusiasmo di Vlaminck alla vista delle figurine dahomeane (o forse wobé), non dimentichiamo che in quell'anno Vlaminck come Brancusi avevano 28 anni, Derain e Franz Marc 24, Picasso 23 e Modigliani 20. Non avevano aspettato poi tanto a lungo. Se i loro predecessori non si erano mai accorti che da secoli esisteva una grande plastica africana, com'è di fatto vero, ciò non faceva che sottolineare la perspicace sensibilità dei giovani.

\section{IL PRAGMATISMO DEGLI ETNOLOGI}

Non si detrae nulla ai meriti dei museografi dell'etnologia affermando che la stessa ampiezza delle loro mansioni incoraggia poco l'adozione di criteri discriminativi fra

I A. A. Gerbrands, Art as an Element of Culture, especially in Negro Africa, Leiden, 1957, pp. 57 sgg.; W. B. Fagg, 'Africane Culture', in Enciclopedia Universale dell'Arte, i, cols. I 33 sgg., Venezia-Roma, 1958. 
le multiformi categorie di oggetti affidati alle loro cure; essa suggerisce più un atteggiamento equanimemente distaccato che non la disposizione a quell'entusiasmo dionisiaco che è alla base dell'apprezzamento estetico. Tutti i musei etnografici contengono collezioni di strumenti musicali e di attrezzi da pesca di ogni parte del mondo; non è detto con questo che i conservatori siano in grado di ricavare e misurare le scale armoniche dei singoli strumenti o di suonarvi sopra le originarie melodie indigene; e su cento direttori di museo, si e no uno saprebbe cacciare le foche con gli arponi artici di cui tutti conoscono i particolari tecnici. Analoghe limitazioni di competenza sono scusabili anche riguardo agli oggetti d'arte.

Ma vi è di più. Per l'etnologo, ogni oggetto etnografico - dal più rudimentale al più perfezionato - ha un suo insostituibile valore e dignità come documento di cultura; ognuno è per lui in certo senso un oggetto d'arte. Si riconoscono certo gradi diversi di abilità tecnologica; ma quanto più l'etnologo è esperto, tanto più la sua valutazione del ' bello' e del ' brutto' è guidata dalla nozione del relativismo culturale, si distacca dai canoni estetici consueti nella sua propria società, diviene dunque mediata e crescentemente complessa.

Teniamoci allo specifico argomento odierno, e ammettiamo per ora di essere d'accordo su quelle che sono (e quelle che non sono) propriamente opere d'arte frammezzo a collezioni africane d'ogni genere e tipo. E dovere dei museologi occuparsi di una varietà di aspetti relativi a tali opere: precisa località di provenienza, data di fabbricazione, identificazione dei materiali usati, funzione degli oggetti nella società di origine, natura dell'eventuale decorazione, etc. Su questi dati analitici si costruiscono quelle che potrebbero dirsi le schede anagrafiche di base della plastica africana, che permettono di passare poi a considerazioni più generali : distribuzione geografica dei centri di produzione artistica nel continente, identificazione dei principali gruppi tipologici, natura dei caratteri distintivi sotto il profilo etnico. L'esame di queste tre categorie di fatti conduce a tre distinti ordini di constatazioni.

Primo, l'esperienza museografica rivela la distribuzione inuguale, sotto l'aspetto geografico, delle produzioni d'arte. P.es., in Africa equatoriale, a occidente della fossa tettonica dei grandi laghi la plastica lignea mostra grande rigoglio e varietà di tipi, mentre a oriente della stessa le forme artistiche mancano quasi del tutto. Si pone con ciò la questione del perché : problema di stretta competenza etnologica.

Secondo, l'uso accertato di un numero sufficientemente alto di singoli esemplari dimostra l'esistenza - su scala regionale o anche continentale - di date categorie funzionali di oggetti a cui va riconosciuto prevalente interesse artistico, quali p.es. tamburi, oggetti divinatori, immagini di antenati e cosi via. L'identificazione selettiva di tali categorie obbedisce a criteri solo in apparenza elementari e ovvi, cioè solo in quanto si dia scontata in partenza la distinzione fra ciò che in Africa è arte e no. Alle maschere, p.es., si suole in genere attribuire un certo pregio artistico, alle pagaie no. Ma una simile distinzione ha carattere del tutto empirico, perché non è insita nella natura strutturale e funzionale della maschera come tale una garanzia di bellezza, né in quella della pagaia la garanzia contraria. Infatti, qua e là nel mondo etnologico esistono tipi di maschere (p.es. irochesi) privi di un vero valore estetico, mentre notoriamente certe pagaie scolpite (p.es. della Melanesia) sono autentici capolavori. Anche la distinzione fra arte e artigianato non può essere stabilita senza riferimenti etnologici. 
In terzo luogo, l'esame dei caratteri formali conduce alla possibilità di stabilire aggruppamenti di oggetti (eventualmente disparati per materia, destinazione ed anche per età di fabbricazione) in cui si riconoscono caratteristiche formali omogenee, dovute al comune rispetto di canoni tradizionali propri di singoli gruppi etnici. $\mathrm{Da}$ ciò deriva l'identificazione di ciò che chiamiamo ' stili'. In un medesimo gruppo stilistico possono includersi opere eterogenee, come sgabelli, pipe, statue, ceramiche, etc.

Gli etnologi hanno seguito diligentemente queste tre vie, assolvendo nei confronti dell'arte africana i primi essenziali doveri : di raccogliere e conservare anzitutto; poi di identificare, classificare e denominare minutamente; infine di individuare certi ordini di nessi formali, funzionali ed etnico-culturali.

\section{T'ENTATIVI DI SINTESI}

$\mathrm{Da}$ questo necessario lavoro analitico, si è poi cercato di passare a una fase di sintesi, come in ogni altro settore della nostra disciplina. E ciò è avvenuto per lo più secondo due diverse direttive.

$\mathrm{Da}$ un lato, si è voluto fissare alcuni canoni di generale validità nella plastica africana : si è così stabilito che essa obbedisce a regole di frontalità e di simmetria, che ha carattere statico più che dinamico, che tende a effetti propriamente plastici e architettonici con la istintiva costruzione di piani e volumi, piuttosto che attraverso l'espressione delle figure; che trascura le proporzioni realistiche; che predilige soggetti isolati più che la composizione di gruppi; e così via. Formule del genere, che costituiscono il nerbo degli scritti sull'argomento, sono vere in via approssimativa; nella misura in cui sono vere, si tratta di semplici constatazioni che non aiutano a capire gran che.

Dall'altro lato, e parallelamente all'affinarsi delle individuazioni stilistiche, si sono intravisti nessi etnico-storici fra i vari gruppi regionali di sculture, ma per lo più senza prove documentate circa le effettive relazioni genetiche, dato che queste (se esistono, come in certi casi è ben possibile) risalgono spesso a epoche su cui mancano attendibili notizie storiche. Già Torday, sulla base di constatate affinità stilistiche, pensava a rapporti fra i Bushongo del Kasai e i centri artistici del basso Congo, da situarsi durante il regno di Alvaro II (I $574-\mathrm{I}_{6} \mathrm{r}_{4}$ ); e p.es. Margot Plass per motivi analoghi, accostando le poco note opere dei BaMbole del Lomami a quelle dei Fang del Gabun, postula la spiegazione delle affinità in una possibile migrazione dei Fang dalla zona dell'alto Congo.I La presenza nell'oreficeria ashanti di simboli zodiacali analoghi a quelli europei (che sono poi d'origine caldea) fa presupporre antichi processi di diffusione trans-sahariana; così come le strette analogie fra certi motivi decorativi malgasci e quelli dei Saadang Toradja o dei monumenti megalitici di Sumba in Indonesia, rilevate da $\mathrm{R}$. Heine-Geldern, suggeriscono remoti rapporti transoceanici. ${ }^{2}$

Ora studi del genere, quando condotti con la necessaria erudizione e con solido metodo, sono di alto interesse: $\mathrm{ma}$ a ben vedere è qui l'arte che fornisce suggerimenti o documenti ai fini delle ricostruzioni storiche, più che non viceversa.

${ }^{I}$ E. Torday, "The Influence of the Kingdom of Kongo on Central Africa', Africa, i, London, 1928, pp. 160 sgg.; M. Plass, African Tribal Sculpture, Phila- delphia, s.d., pp. 55-56.

${ }^{2}$ R. Heine-Geldern, 'Indonesiane Culture', in Enciclopedia Universale dell'Arte, vii (in stampa). 
E un fatto, soprattutto, è importante notare: le analisi e indagini fin qui accennate si basano esclusivamente sui caratteri esteriori dell'arte che studiamo. Ora il primo presupposto per poter dire qualcosa di valido sul contenuto di quest'arte, e per risalire di qui fino alla sua essenza profonda e al suo significato, è formarsi idee precise sulla sua funzione sociale e spirituale. $\mathrm{Da}$ questo traguardo, come ben sa ogni etnologo, siamo ancora ben lontani. Vi sono alcune fortunate eccezioni-e fra queste vorrei citare lo splendido studio di M. Griaule sulle maschere dogon - ma la stragrande maggioranza dei pezzi nei nostri musei è stata raccolta senza il necessario corredo di notizie al riguardo.

In via superficiale, certi oggetti considerati d'arte sembrano offrire nella loro ovvia funzionalità la spiegazione richiesta. Uno sgabello scolpito servirà per sedercisi sopra, $\mathrm{da}$ un tamburo si ricaverà suono e ritmo. Ma per quale ragione un dato sedile luba ha il piano d'appoggio sorretto da una cariatide femminile? per puro gusto ornamentale? per commemorare un'antenata? per dare simbolo tangibile a un culto della fertilità? o semplicemente per dare espressione alla sensualità dello scultore? E perché quel tale tamburo reca una figura di lucertola in bassorilievo? si tratta di un emblema araldico, di un totem, di un'allusione allegorica, o di una gratuita risorsa decorativa? e quella figura è o fu scolpita per il diletto estetico o per le esigenze simbolicoreligiose dell'artista, o del suonatore, o dei ballerini e ascoltatori, o di un dato dignitario o committente, o di tutti insieme? E cosi via.

Quando poi, a differenza da questi casi, l'opera d'arte non ha una funzione concreta direttamente intuibile, il suo significato può dar luogo a incertezze totali. In passato, ogni statuetta africana era semplicisticamente battezzata in Europa un 'idolo'; quando divenne evidente che nessun Africano idolatrava statue di sorta, il termine fu sostituito con quello meno compromettente di 'feticcio' oppure quando neppure un uso magico poteva essere sostenuto - con il nome 'figurina di antenato'. Ma bisogna avere il coraggio di riconoscere - cito qui il miglior conoscitore della plastica congolese, F. Olbrechts - che anche quest'ultimo termine non ha spesso altra ragion d'essere che quella di mascherare la nostra ignoranza. ${ }^{\mathrm{x}}$

In anni recenti, con il diffondersi della cultura etnologica, alcune collezioni sono state formate da studiosi preparati, e - quando le circostanze erano favorevoli - le informazioni sono divenute più precise, se non esaurienti. Ma si tratta sempre di eccezioni. Per il $90 \%$ degli oggetti di museo, la funzione deve essere ricostruita congetturalmente a posteriori, sulla base di analogie esteriori con opere consimili di cui si abbiano più fortunati ragguagli.

In assenza di dati sufficienti a una comprensione precisa, si suole attribuire alla scultura africana una generica funzione religioso-sociale, a un dipresso come la si attribuisce alla scultura medioevale, ma con minor conoscenza di causa. Se un'opera ci appare paurosa o mostruosa, la sua genesi si spiega con presunti sentimenti di crudeltà o di terrore nell'artefice che l'ha creata. L'espressione ritenuta feroce di una statuetta 'magica' è interpretata come esprimente la malignità che lo scultore attribuisce - intenzionalmente o subconsciamente - all'essere raffigurato; o allo stato d'animo di chi è (o si crede) in lotta contro le insidie oscure del sortilegio, della carestia, della morte. Tutto ciò, s'intende, è abbastanza vago e arbitrario. Certe espressioni che definiamo fisse, elusive, enigmatiche, le giustifichiamo con la natura

I F. M. Olbrechts, Les Arts plastiques du Congo Belge, Bruxelles, I959, p. I05. 
insondabile e misteriosa degli esseri soprannaturali rappresentati, la cui azione oracolare è impredicibile. Ma se uno sguardo parimenti vacuo lo riscontriamo in una maschera, la spiegazione prefabbricata è che essa andrebbe vista non nell'immobilità della vetrina, ma nell'azione pantomimica e drammatica a cui fu destinata e da cui attinge il latente dinamismo.

Può esservi del vero in simili tentativi di interpretazione che soddisfano la nostra razionalità, ma essi non spezzano ancora il circolo del nostro etnocentrismo: ci indicano soltanto la via giusta per uscirne.

Lo stesso può dirsi per certe generali considerazioni suggerite dalla retta tendenza etnologica a considerare l'arte (come ogni altro aspetto della cultura) in relazione al quadro totale: quali, per citare le più note, che la scultura lignea è più diffusa, e di più alto livello, fra le società di coltivatori che fra quelle di pastori; che si accompagna a sistemi di discendenza matrilineare più spesso che non a quelli patrilineari; che raggiunge le massime fioriture presso le corti aristocratiche e nei centri tradizionali detti di tipo ' urbano'. Si tratta di generalizzazioni aventi una certa validità statistica, e non certo di leggi sociologiche o economiche di validità assoluta; e se ne possono dare spiegazioni abbastanza logiche. Ma quando si tenti di ridurle a formulazioni più precise - p.es. di collegare la staticità stilistica con sistemi sociali rigidi, e viceversa - il tentativo, lo ha notato anche un funzionalista come R. Firth, ${ }^{,}$si risolve in una vaga previsione di probabilità. Una impostazione sociologica della ricerca è utile $\mathrm{e}$ legittima, ma non sufficiente alla piena comprensione del fenomeno artistico.

\section{UNA DUPLICE 'Presa di COSCIENZA'}

Poiché in sostanza, a che cosa mirano e devono mirare gli studi su questa come su ogni altra grande categoria di arti ? Ecco una domanda a cui è tempo di rispondere prima di cercare una conclusione al nostro discorso.

La prima ovvia esigenza è beninteso una conoscenza del fenomeno in tutti i suoi possibili aspetti; e a questo fine mirano gli studi nelle diverse direzioni accennate. $\mathrm{Ma}$ a quali fini ulteriori deve servire questa conoscenza? Essere informati su una qualsiasi categoria di opere d'arte non è fine a se stesso; vogliamo poterle apprezzare; il che significa insieme intenderle e amarle, perché solo così il nostro rapporto umano con esse porta a un arricchimento del nostro spirito. Sorge cosi inevitabilmente il problema del ' bello' nelle sue vecchie alternative di obiettività e subiettività. ${ }^{2}$ Nessuno meglio dell'etnologo sa fino a qual punto l'apprezzamento estetico sia facoltà culturalmente determinata, vale a dire quanto sia vero che la bellezza è nell'occhio di chi guarda più che nella cosa guardata.

Ora quando ci poniamo di fronte alla ceramica etrusca, all'architettura maya, o anche alla pittura fiorentina del Quattrocento, ne rievochiamo i valori sul metro della nostra sensibilità moderna e possiamo tener conto della nostra sola emozione estetica. Ma il fenomeno dell'arte africana è molto vicino a noi nel tempo, patrimonio di un'umanità viva che non si è ancora rassegnata a staccarsene consegnandolo agli archivi chiusi del passato. Siamo in due a vedere. E, per dirla con Roland Colin, ' non

${ }^{I}$ R. Firth, Elements of Social Organization, London, I95I, p. 170 .

${ }_{2} \mathrm{Si}$ vedano al proposito le osservazioni di $\mathrm{H}$. Schneider, 'The Interpretation of Pakot Visual Art',
Man, Ivi (1956), n. I08, e i commenti di M. J. Herskovits, 'Art and Value', in Aspects of Primitive Art, New York, 1959, pp. 44 sgg. 
si tratta, per il Negro o per il Bianco, di vedere da soli, anche se si vede bene. Importa, anzitutto, vedere insieme' '.

In tale senso, il nostro studio, più che una semplice esegesi storico-critica, deve essere una duplice presa di coscienza, a cui l'umanità consapevole dell'Africa porti il suo contributo diretto. Indirettamente, si noti, ciò è già stato avviato, poiché negli studi migliori sull'arte africana l'etnologo europeo o americano è stato a volte fedele portavoce dell'informatore africano o (come nei casi di Himmelheber, di Vandenhoute, di Griaule) dello stesso artista creatore. Si tratta, ripeto, di passare dal contributo indiretto a quello diretto: per questo, speriamo, i tempi stanno maturando.

Una collaborazione del genere potrà intanto aiutarci a impostare più razionalmente, se non a risolvere, il problema della discriminazione fra arte e artigianato in Africa. In quello che è il più serio contributo degli ultimi anni sul nostro argomento, A. A. Gerbrands ha proposto la seguente 'definizione etnologica' dell'arte: 'Se un individuo creativo dà a certi valori culturali un'interpretazione personale tradotta in materia, movimento o suono, di natura tale che le forme risultanti da questo processo si adeguano ai criteri di bellezza validi nella sua società, chiamiamo questo processo creativo, e le forme che ne risultano " arte ".'z .

Su questa definizione, che certo è maturamente meditata, io avrei qualche riserva da fare in altra sede (così com'è formulata, essa suona eccessiva abdicazione del critico occidentale, una sua rinuncia a considerar validi ogni apprezzamento ed emozione nostri). Ma l'ho citata per dimostrare che nell'impostazione etnologica più moderna si dà importanza fondamentale (anzi, qui, esclusiva) ai giudizi di valore delle singole società in cui le singole forme d'arte vengono create. Si tratta ora, dove siamo ancora in tempo, di accertare quali siano tali giudizi, in base a quali valori siano formulati ed espressi. È un'impresa nobile e impegnativa, diciamo pure molto ardua. Dobbiamo aggiungere 'utopistica'?

Molto dipenderà dall'atteggiamento futuro degli Africani. In passato, i loro artisti hanno saputo creare mondi loro di forme e valori estetici entro cerchie relativamente chiuse, che non favorivano una consapevolezza critica d'ampio respiro; bene o male, il tentativo di integrare quelle produzioni artistiche nel patrimonio spirituale dell'umanità, è stata opera europea. Questa non concordata ripartizione di compiti è finita, o sta per finire.

Ora il riavvicinamento degli Africani, con mutato spirito di consapevolezza, al loro recente passato creativo, avviene tardi, quando le loro arti secolari sono entrate in una crisi che tutto lascia supporre definitiva. Tale ritardo non è casuale, ma determinato da quella che una volta tanto ci si permetterà di definire una fatalità storica. Poiché se è vero che la decadenza repentina della scultura negra è conseguenza dei violenti scompensi spirituali e sociali dovuti all'acculturazione, non è meno vero che a quest'ultima gli Africani devono l'essere progrediti, sia pure con élites finora esigue, a un livello letterato di civiltà. E proprio con l'assumere certe forme della civiltà occidentale essi cominciano ad acquistare una prospettiva storica delle loro passate creazioni non più sul piano limitato della tribù o nazione, ma su quello più universale dei valori artistici mondiali.

Non del tutto logico, se pur comprensibile, è l'atteggiamento di protesta polemica

I R. Colin, 'Situation de l'art nègre', Présence Africaine, N.s., no. 26 , juin-juillet 1959 , p. 57.

\footnotetext{
${ }^{2}$ Gerbrands, op. cit., 139. La sottolineatura è mia.
} 
con cui si manifesta il tardivo intervento di certi intellettuali africani in questo campo, che più che da interessi artistici par derivare da una posizione di antagonismo e di rivendicazione politica. Il nocciolo di tali argomenti è espresso nello scritto recente di un influente scultore nigeriano: "The science of anthropology has ... been used to create an intellectual barrier which makes it extremely difficult for most Africans to be considered qualified to play an important part in the development and preservation of their native art. . . While Europeans are the best judges of their own art, and no one argues about this fact, the African does not even have a chance to play an equally important part in judging his art, let alone his justifiable claim ... that he is the best judge of his own art. ... Outside his country today, he faces the humiliation of having to listen to lectures on African art in foreign art galleries and museums. He visits foreign museums in order to see a collection of the art of his own country, and very often he is shown round the museum by European curators. This aspect of the problem is cultural, emotional, and political.'.

Intendo deliberatamente lasciare fuori del quadro l'aspetto politico, perché ritengo che serva solo a complicare indebitamente, non a chiarire i nostri problemi. E fra questi, non intendo toccare quello - pur importante - dell'avvenire dell'arte africana, perché, come direbbe Kipling, questa è un'altra storia. Ma nella valutazione del passato e presente di quell'arte, gli aspetti 'culturali ed emotivi' cui allude Ben Enwonwu sono certo da tenersi presenti.

Si potrebbe rispondergli, beninteso, che fra tanti aspetti discutibili dei rapporti euro-africani negli ultimi secoli uno dei più genuini (se non sempre dei più disinteressati) è rappresentato proprio dalla passione dei Bianchi per l'arte negra; che essa ha valso, quanto meno, a salvare dalla distruzione infinite opere altamente deperibili che negli ambienti d'origine non si sarebbero conservate, e che oggi sono a disposizione di tutti gli uomini della terra; che è merito e non colpa dei Bianchi se essi hanno preceduto gli Africani nel culto e nello studio delle tradizioni artistiche antiche e recenti, loro proprie e altrui; e che in tal modo, e solo così, la scultura africana ha conquistato - com'egli del resto ammette - un posto d'onore fra le arti del mondo intero. Si potrebbe anche ricordargli questo: che a parità di cultura generale e di erudizione con lo studioso di razza bianca un Yoruba, poniamo, avrebbe in partenza migliori titoli per un'analisi approfondita dell'arte yoruba o (forse) nigeriana in genere, ma per tutto il resto il suo compito non sarebbe meno arduo di quello dei suoi colleghi europei, a cui si deve almeno il primo tentativo di inquadrare la scultura yoruba nell'orizzonte dell'arte universale.

Possiamo certo rispondere tutto questo; ma non possiamo fermarci qui. Le rivendicazioni della intellighentsia africana valgono in effetti ad aggiungere un'altra esigenza fra quelle di cui già noi stessi eravamo consci, e a farci riproporre il problema non mai risolto dell'equa valutazione di arti che sospettiamo essere in fondo state, se non fraintese, almeno inadeguatamente intese. Poiché, per diligente e appassionato che sia stato finora il nostro studio, esso rimarrà incompleto fino a che ci limiteremo ad applicare, nell'apprezzamento di creazioni esotiche, i soli criteri estetici del nostro proprio mondo culturale.

Ciò non vuol dire che dobbiamo farci negri per poter intendere la scultura negra,

\footnotetext{
I Ben Enwonwu, 'Problems of the African Artist Today', Présence Africaine, N.s., nos. 8-Io, juinnovembre 1956 , pp. $177-8$.
} 
così come non è necessario essere uomini del Duecento per intendere Duccio o Cimabue; ma vuol dire che dobbiamo immedesimarci nei valori spirituali, storici, sociali ed estetici delle culture africane con lo stesso impegno critico e con la stessa partecipazione intellettuale e pienamente umana, con cui ci accostiamo alle fasi di civiltà del nostro passato occidentale.

\section{L'ESIGENZA DI UN'ANALISI CRITICO-ESTETICA INTEGRALE}

Ricapitoliamo allora brevemente i risultati che fin qui noi Europei abbiamo, per conto nostro, raggiunto nell'avvicinarci alla plastica africana: in attesa di metterli a raffronto e a integrarli, quando il momento opportuno verrà, con i contributi critici sostanziali che ci verranno dall'Africa stessa.

Salvo le debite eccezioni, la generale debolezza del gigantesco lavoro compiuto specie nel XX secolo dagli esegeti europei dell'arte africana consiste nel fatto che questa è stata esaminata più sotto il profilo della forma che sotto quello del contenuto. Debolezza a volte programmatica, perché anche i più colti storici d'arte, come Eckart von Sydow, proclamavano: "when examining primitive works of art, the right method is to concentrate on the analysis of formal structure as such'. ${ }^{\text {I Si tratta }}$ di una debolezza oggettiva, perché la plastica africana al pari delle altre arti c.d. 'primitive' d'ogni livello luogo ed epoca ha questo se non altro in comune con la scultura europea tradizionale, di presentare un nesso insopprimibile fra forma e contenuto: l'aspirazione a un'arte 'pura' come l'intendono certi nostri contemporanei è sconosciuta a questi livelli.

A questo riguardo, le interpretazioni degli artisti ed esteti nostrani sono molto scadenti, come lo dimostra sul piano letterario il fiume di scritti di questa fonte che ben conosciamo, e che continua a scorrere ancor oggi copioso. I Fauves ebbero, come s'è detto, il merito non piccolo di avere intuito per tempo il valore estetico della scultura africana; ma la totale ignoranza del suo significato originario precluse loro ogni possibilità di vera comprensione. Se sul puro piano formale i cubisti ne apprezzavano legittimamente i valori architettonici evidenti, gli espressionisti si ponevano in un vicolo cieco esaltando in quell'arte un contenuto mistico-emotivo di cui nulla sapevano. Non sta all'etnologo giudicare i frutti buoni o cattivi (io credo cattivi) dati da una pianta di così mal fondate radici; ma il buon senso suggerisce che in arte, come in politica e in altri campi, è sconsigliabile lasciarsi influenzare da ciò che non si capisce. E mi astengo dal parlare di certe valutazioni ispirate a criteri psicoanalitici, le quali suggeriscono per lo più commenti ancor meno benevoli.

Quanto hanno prodotto gli etnologi è naturalmente di molto maggiore importanza; essi hanno dato, se non altro, una classificazione degli stili e sottostili tribali che rimane una solida base per ogni futura indagine. Ma se dagli etnologi è legittimo attendersi dottrina e competenza tecnica, non altrettanto necessario è che essi palesino sensibilità estetica e buon gusto; la mancanza di apprezzamenti può anzi essere voluta, il compito specifico dell'etnologo essendo quello di fornire gli elementi tecnologici, iconografici, storici etc. che sono il presupposto della valutazione estetica, e non già di formulare giudizi di valore.

In breve, dunque, il critico d'arte non può fare qui valida opera storica perché

' E. von Sydow, 'African Sculpture', Africa, i, London, I928, p. 21 I. 
gli mancano le premesse e le cognizioni degli etnologi; e l'etnologo non è sufficientemente attrezzato di nozioni storico-artistiche per fare valida opera critica. ${ }^{1} \mathrm{La}$ subiettività di giudizio del primo si rivela pericolosamente fallace su un terreno tanto diverso da quello su cui tradizionalmente si esercita la critica estetica; mentre nel secondo lo habitus mentale della distaccata obiettività ostacola quella speciale sensibilità (o intuizione sintetica come la chiama Panofsky) che è alla base dell'emozione estetica.

Ciascuno dei due vede dunque del fatto artistico africano un lato che all'altro rimane oscuro; e nessuna delle due metà del quadro acquista il suo significato se non in funzione dell'altra. La conclusione che si deve trarre da ciò mi pare molto elementare, benché non mi risulta sia mai stata esposta in tutte lettere: l'arte noneuropea di livello tribale potrà essere rettamente intesa e valutata solo da uno studioso il quale padroneggi a pieno i due ordini di conoscenze, quello storicoartistico generale e quello etnologico. Poiché è inverosimile che uno storico dell'arte riesca ad abbracciare una scienza così vasta e complessa come l'etnologia, dovrà dunque essere l'etnologo a compiere lo sforzo. Alcuni fra noi si sono in certo modo già posti, difatti, su quella via: ma nessuno, credo, con sistematica preparazione. Si tratta, come ognuno intende, di impresa sommamente impegnativa. Se essa fosse un giorno affrontata con successo da un Africano, che precedesse su questa via $i$ colleghi europei e americani, avremmo da rallegrarcene grandemente. Ma sarebbe beninteso ingenuo pensare che la sola qualifica di una qualsiasi cittadinanza africana basterebbe a ridurre per l'eventuale protagunista dell'esperimento le difficoltà di questo, se non per la parte minima riguardante la sua propria arte tribale.

La conclusione di un discorso necessariamente generico tende per fatalità a lasciare - con una bella frase finale - le cose come sono. Vorrei invece in questa occasione chiudere le mie parole con un riferimento concreto.

Giudicando un recente e pur dotto libro belga sulla scultura pende, G. I. Jones si rammaricava qualche mese fa che tante monografie contemporanee insistessero a farci vedere ' la maschera, e non l'uomo e la società dietro di essa '. ' After reading contemporary monographs on the sculpture of an African tribe one is still looking at a number of masks hanging in a glass case; all one has learnt is where they come from and how to distinguish them from a number of other masks hanging in a number of other cases."2 Ora applicando allo studio delle maschere e scettri pende, o della statuaria luba, o di qualsiasi altro gruppo di plastiche africane, i criteri moderni della critica estetica, lo studioso non rischierebbe più d'incorrere in simili rimproveri. Penso in particolare al metodo suggerito da Erwin Panofsky per lo studio dei problemi iconografici e iconologici in generale, nella sua Introduzione allo studio dell'arte rinascimentale. ${ }^{3}$

La prima fase dell'indagine, che Panofsky chiama analisi pseudo-formale o descrizione pre-iconografica, verte sull'argomento o soggetto immediatamente visibile; si occupa del tipo di rappresentazione com'è manifestato a mezzo di linee, piani, volumi e colori, ossia del mondo delle pure forme, riconoscibili come portatrici di significati 'naturali' o primati. Dalla serie dei 'motivi' si risale, con l'ausilio dell'esperienza

I Cfr. A. Schweeger-Hefel, Holzplastik in Afrika, Wien, $1960, \mathrm{p} . \mathrm{I}$.

${ }^{2}$ Africa, xxxi, 196I, p. 98.

${ }^{3}$ E. Panofsky, Meaning in the Visual Arts, Garden City, N.Y., 1955, pp. 26-54. 
pratica, all'identificazione degli stili o scuole: è, come si vede, la fase a cui molto spesso i museografi dell'etnologia si sono arrestati.

La seconda fase, o analisi propriamente iconografica, si occupa dei soggetti secondari o convenzionali, di non immediata evidenza, ossia delle immagini, storie e allegorie. Essa mira a stabilire, intrinsecamente e comparativamente, il significato spesso recondito dell'opera d'arte, o che è palese solo nel luogo e periodo in cui essa è prodotta a meno che non intervenga un elemento esoterico. Qui già si manifesta una decisa differenza nelle tecniche di interpretazione, offerte nel mondo culto dalla conoscenza delle fonti letterarie (da cui si trae la 'dimestichezza con specifici temi e concetti '), mentre nella sfera etnologica esse deriveranno da un'investigazione in profondità della tradizione orale e dall'attenta ricognizione etnografica. Questa avrebbe però il vantaggio di potersi spesso limitare a una analisi locale, perché (a differenza da quanto avviene nella nostra civiltà, ove ampia è la circolazione di temi e immagini da un paese all'altro attraverso tradizioni dotte aventi radici nella mitologia classica, in soggetti biblici o storici, etc.) accade che ogni mondo tribale sia concluso o quasi completo in sé, con un patrimonio ideale più limitato e meno rapidamente variabile nel tempo, oltre che condiviso da gran parte dei membri adulti della società. A questo secondo stadio, che dovrebbe darci la piena comprensione della tematica, noi siamo giunti finora solo in minima parte.

Solo nella terza fase, della interpretazione iconologica, si giunge però ad affrontare il contenuto o significato 'intrinseco ' dell'opera d'arte, che Panofsky dice costituire il mondo dei valori simbolici. Si tratta, secondo lui, di comprendere perché l'artista si sia servito, per esprimersi, di quei determinati temi e concetti che sono alla base delle sue concrete produzioni; e perché, avendoli scelti, li ha presentati in un dato modo proprio alla sua personalità oltre che alla sua cultura e alla sua scuola. E possibile che in questa terza e più delicata fase qualche cosa si debba modificare e sostituire nelle modalità interpretative di Panofsky trasposte sul livello così diverso delle arti africane: la dimestichezza con le tendenze essenziali della mente umana è forse uno strumento d'interpretazione troppo vago, anche se temperato dagli accorgimenti e controlli che egli prevede. Rimane tuttavia vero che è proprio quel ' modo' difficilmente definibile della singola espressione artistica, legato al genio individuale del suo autore, che ci procura l'emozione estetica, non già il soggetto stesso figurato né i suoi sottintesi allegorici - a qualsiasi mondo culturale essi appartengano, nostrano o remoto.

E rimane vero che proprio a quell'elemento intuitivo e irrazionale, che si rifiuta di lasciarsi costringere in regole e metodi nel momento dell'interpretazione come in quello creativo, noi dobbiamo i singolari incontri degli spiriti umani oltre le barriere degli stili e dei continenti; e intravediamo un altro e più vasto problema, quello dei valori universali dell'arte.

\section{Summary}

\section{MEANING IN AFRICAN SCULPTURE}

THE knowledge of African sculpture has had a paradoxical history. Over the centuries when exoticism was fashionable in Europe, this exotic art par excellence was totally ignored; it became famous, however, the moment exoticism in its refined (Eastern-inspired) forms started to decline. Its finest examples were collected when few realized their intrinsic value; 
they later attained exaggerated tenown, thanks to persons who knew practically nothing about them, and were praised for just those merits which they did not possess. Yet at the same time that Negro sculpture contributed a vital-though improperly used-leaven to the mass of Western art, Europeans were bringing about its complete collapse. Finallyand even more to be regretted-Africans are now acquiring a vicarious consciousness of their art traditions just at the time when it seems that only a miracle can preserve them.

The cultural, social, political, and economic reasons for these apparent contradictions, though having only an indirect connexion with art, are well known. I shall therefore confine myself to the art-historical aspects of the problem.

The fortune of African sculpture in Europe began in or around 1904. Everybody is now familiar with the story of how Maurice de Vlaminck took a fancy to certain figurines (Dahomean, it is said), discovered on the shelves of an Argenteuil bistro, and bought them for a few francs; he then gave one to Derain, which was to arouse the lyrical enthusiasm of Guillaume Apollinaire and later of Pablo Picasso.

I have deliberately said 'fortune ' rather than ' discovery' of African sculpture, because the real discovery of this art dates back to far remoter times. Since the end of the fifteenth century, Portuguese seamen had revealed to the West the striking works in bronze, wood, and ivory of the Guinea coast. This unsuspected skill of African artists caused Europeans to stimulate a 'commissioned' production which was much sought after in European courts : artists from Guinea were possibly active in Portugal in the sixteenth century. Be this as it may, we have here one of the first known phenomena of partial Euro-African acculturation, the most famous examples of which are the ivory ladles, hunting-horns, and bowls (labelled as Benin even if they originated in other centres of the Guinea coast), several of which are preserved to this day in our museums. Appreciated as curios, they did not arouse -as far as we know-any impulse among European artists of the day, who were engrossed in ideals and models of an altogether different sphere.

For over three centuries Africa supplied Western art with no more than occasional hints on local colour and costume, and moreover these were Moorish rather than African. In the second half of the nineteenth century, and the first two or three decades of the twentieth, large ethnographic collections were assembled, though at first in a haphazard way, from all parts of the continent, occasionally including those elements which were later to be acknowledged as ' works of art'. At the same time, European penetration was beginning to upset the indigenous balance of African societies, ousting ancient techniques by introducing new ones, and undermining systems of beliefs in which native craftsmen had found their inspiration. Between the end of the nineteenth and the beginning of the twentieth century, two concurrent processes thus took place which were to remain fundamental for all future developments. On the one hand, a major (in some cases the best) part of African plastic production was transferred from its homeland to collections and museums in Europe and America. On the other hand, almost everywhere the creative centres of those arts declined or disappeared, or were degraded to centres of mediocre craftsmanship of ' tourist ' commercial type.

Today-in $196 \mathrm{I}$-the typological inventory of African sculpture is almost complete and final. Hopes of still finding some uncontaminated area of artistic production must be abandoned: Africa has no more unexplored peoples or 'corners'; in this field there is nothing more to 'discover'. Her art is being studied in handbooks, monographs, or in museum glass cases, like that of extinct or buried civilizations; and, but for a few exceptions, this study has the anatomical character of an autopsy rather than that of a physiological analysis on a living organism.

A preliminary remark which must be made here is of major importance, though-or, rather, because-we may find it natural or self-evident. Almost all evaluations of these art 
forms have hitherto been made by Europeans (or Americans) without paying any attention to the criteria and judgements of Africans themselves. Reasons for this may be three. Either the reactions of Africans concerned were not, and could no longer be, known-as in the case of objects of uncertain origin or collected at an early date; or it was thought that the Africans' opinions on the subject were irrelevant on principle; or it was supposed that their appreciation, if it were known, would necessarily coincide with that of Europeans, in keeping with a presumed universality of aesthetic values-a strange idea, since the profound differences between Negro and European arts should have led one to surmise exactly the contrary.

At any rate, reactions of white people to African arts must be divided into two fairly clear-cut categories: those of aesthetes, men of letters, and artists on one side, those of anthropologists on the other. The reactions of the average public constitute a third category, which may here be ignored, not because it reflects less significant opinions (indeed, these are probably more genuine and unprejudiced) but because it is more uncertain and difficult to control, being anonymous in nature and seldom recorded in writing.

The ideological programme of Fauvism, which sprang up in violent reaction to the conventionalism of 'civilization', included among other things a re-evaluation of 'primitives', in which the Fauves would see, rightly or wrongly, spontaneity of expression and vehemence and daring in the use of violent colour and bold form. Just as a character of Andre Gide confessed in those years that he was " seeking in books the justification of his actions ', so the Fauves considered that they had found in Negro sculpture an ante litteram justification of their new tendencies and aesthetic credo. Their indifference to the original significance of that sculpture equalled that already shown by Gauguin towards Polynesian art.

Their enthusiasm for Negro art coincided with their rejection of aesthetic principles established since the days of the Renaissance, and thus far unchallenged. German expressionists followed similar trends when they sought inspiration in what they took to be the aboriginal purity of the savages and praised them for their love of abstraction. Stress was laid on form, but artists could hardly resist the temptation of attributing to the 'primitives' intentions and emotions which were largely guesswork, thus shifting their interest imperceptibly from form to content. From different points of view, scholars like Lévy-Bruhl, writers like Gide, and even Africanists like Delafosse, all writing in the twenties, were in agreement when they depicted the African as guided exclusively by such forces as mysticism, irrationalism, and collectivism. Whatever their intentions when imitating (or being 'influenced' by) African models, such artists as Lipschitz and Klee, Moore and Modigliani, were following a source of inspiration of which they could not but misunderstand the true values.

It is not for the ethnologist as such to pass judgements on the success achieved by Western artists in their attempt to integrate African elements into their own paintings and sculptures. However, he is entitled to state the general principle that no positive and constructive process of acculturation can be achieved unless it is based on mutual cultural understanding as a prerequisite of any integration process. The ethnologist is further in a position to point out that the alleged primitivism of African sculpture, by which a number of Western artists were ostensibly influenced, was and is a misleading source of inspiration, in that African art cannot be termed primitive at all.

In spite of the naivety detected by anthropologists in these reactions, the sudden outburst of enthusiasm by artists, most of them very young, for African sculpture in the early years of our century was no doubt spontaneous and has had a profound significance in our own culture. A similar sensitivity and response was hardly to be expected of museum keepers. The enormous variety of objects entrusted to their care and study makes for an impartial attitude of detachment rather than for an inclination to the dionysian enthusiasm which is 
at the root of aesthetic appreciation. Furthermore, any ethnographic object, whether coarse or refined, has its own claim to the keeper's attention. Degrees of technological skill are no doubt detected; but the more the ethnologist is a scholar in his own right, the more his judgement of 'beautiful' and 'ugly' is guided by the notion of cultural relativity, and therefore detached from the aesthetic criteria of the society to which he belongs.

Even granted that the museum ethnographer should be in a position to make a clear-cut distinction between what is, and what is not, an art object, his first duty towards the former class is manifold. Exact place of origin, date of manufacture, identification of materials used, function of the object in the society it comes from, nature of its decoration, \&c. are among the main things to be assessed. Analytical data of this type collected on a large number of ' art' objects lead to more general considerations, such as the geographical distribution of art-producing centres and areas throughout the continent, identification of the main typological groups and of distinctive ethnic characters. Each of these classes of problems leads in turn to distinct orders of knowledge, particularly significant being the establishment of 'styles' based on traditional canons of form followed by given ethnic groups.

Attempts to pass from an analytic to a synthetic type of study have mainly followed two trends. One has been the tentative formulation of 'laws' valid in African sculpture in general (such as, for example, its tendency to frontality and symmetry, disregard of realistic proportions, static character, and many more). This has led to the creation of a jargon widely used in most publications on African art but of no great avail for its understanding. The other trend is comparative and ultimately historical in nature, and consists in frequent attempts to correlate sculptural styles as wholes often at great geographical distance, or decorative motifs and the like, suggesting past ethnic contacts or relations. Studies of this type can be useful when based on reliable evidence, but even so they constitute a contribution to historical reconstruction, using art elements as evidential materials, rather than a use of ethno-historical materials in the service of art history.

All these various types of study have one common feature, i.e. they are almost exclusively based on the exterior or formal aspects of African art. In order to say something relevant to the understanding of its essence and meaning, we should be clear as to its social and spiritual function. In this respect, with a few notable exceptions, we are still far from the goal. In a superficial way, some objects reckoned as 'artistic' (e.g. drums or stools) are self-indicative of their obvious function, but this does not of course provide the reason for the aesthetic quality in their lines, proportions, or decoration, for the meaning of these and for the reason and purpose of their being ' beautiful '-for the benefit and pleasure of whom? In other cases, when function is not immediately obvious (as in free statuary), we are often at a loss : the vague interpretation of what used to be called 'idols ', then 'fetishes', and now 'ancestor figures' in most of our museums is a well-known example. There is some justification in generically attributing a magico-religious function to African sculpture, but when it comes to specific interpretation of single works of art the Western critic's explanation often suffers from ethnocentrism. Tendencies to place and explain art productions in the general framework of whole cultures are also fully justified, but have so far yielded minor results towards an understanding of the artistic phenomenon.

At this stage we should clearly state the aims of our study. The first aim is naturally the widest possible range of knowledge, and much has been and is being done in this direction. But what are the further aims of this knowledge? To be fully informed on a work of art is not the final end, we want to be able to appreciate it, i.e. at the same time to understand and enjoy it. Here the age-old problem of the concept of ' beautiful', in its subjective-objective alternative, can no longer be avoided. No one is better aware than the ethnologist of how far aesthetic appreciation is a culturally determined faculty. Now when we turn to such art forms of the past as Etruscan pottery, Maya architecture, or Florentine painting of the 
Quattrocento, we may be justified in relying on our modern sensitivity, i.e. on our own aesthetic emotions. But African sculpture belongs to a living humanity that has not yet resigned itself to 'deliver' it to the archives of a dead past. There are two of us looking at it-white man and black man-and what matters, as R. Colin has put it, is to be able to 'see together'. Our aim, then, is a double prise de conscience, and the contribution of Africans is needed.

Indirectly, steps have been taken in this direction. In some of the best studies (such as those of Griaule, Vandenhoute, and Himmelheber) the Western critic has tried to be the interpreter or mouthpiece of the African informant, indeed of the artist himself. Gerbrands has gone so far as to state that the criteria of beauty which must guide us are those valid in the society of the artist. If this were taken literally, it would mean the final abdication of the Western critic. Nevertheless, there is an urgent need to know more about African judgements of their own art and about the values on which they rest. In the recent past, African artists succeeded in creating their own worlds of aesthetic forms and values in closed tribal circles. It was the task of Europeans to try to integrate these artistic productions into a world-wide sphere, to introduce them into the spiritual heritage of mankind. The separation of these two tasks is now nearing its end.

Africans are coming late to a new and broader appreciation of their own creations. The crisis in their arts is a result of Western acculturation, but so is the new consciousness and sense of historical perspective they have acquired which enables them for the first time to view their works of art not only at a tribal level, but on the plane of world artistic values. Some African intellectuals have expressed dissatisfaction, or even a feeling of resentment and 'humiliation', in face of the part played by Europeans in collecting and judging their sculpture. This attitude shows some logical inconsistencies and calls for legitimate answers on our part. But on the other hand we cannot content ourselves with a one-sided defence of the role we have played so far. Western scholars may well pride themselves in having for generations devoted their loving care, their learning and insight, to the preservation of a wealth of exotic art treasures that might well otherwise have been lost for ever to the whole world, including modern Africans themselves. But it is clear that in the appreciation of this wealth we are no longer justified in relying only on the aesthetic criteria of our own cultural environment. The collaboration of learned Africans is vital to our studies if these are to progress. For our part, it is high time we devoted ourselves to the spiritual, historical, social, and aesthetic values of African cultures with the same intellectual concern with which we approach the phases of our Western civilization.

The moment we do so, we realize that 'to concentrate on the analysis of formal structure as such', as von Sydow advocated, is not enough : in African sculpture, as in all arts improperly termed ' primitive', form is inseparably linked with meaning, 'pure' art as understood in some contemporary circles being unknown at these levels. We must further admit that interpretations given in this field by our artists and aestheticians are as a rule lacking in significance or even false. The contributions of ethnologists include much of lasting usefulness, but they seldom approach completeness. The art critic is unable to do any solid arthistorical work in this field, because he lacks ethnological knowledge, whereas the ethnologist is not sufficiently equipped with a background in art criticism to express valid judgements. Each one sees one side of the picture which remains hidden from the other. But as only a full view of the phenomenon can be satisfactory, the obvious answer to this antinomy is that non-European tribal art will have a chance of being rightly understood and appreciated only by scholars mastering both fields of knowledge.

The type of studies that could and should be achieved in regard to Negro sculpture will be realized if we consider the methodological requirements stated, for example, by Erwin Panofsky for the analysis of Renaissance art. A first stage of the study, which he calls pre- 
iconographical description (and pseudo-formal analysis), is concerned with the primary subject-matter, i.e. in our case with the sculptures as such-the world of pure forms and volumes and of their apparent meanings. This leads in turn to the identification of styles or schools-the stage at which most monographs on African sculpture have stopped. A second stage, described as iconographical analysis proper, deals with the "secondary or conventional subject matter, constituting the world of images, stories and allegories'. At this stage, techniques of interpretation must needs vary in our special field, on account of the lack of literary sources. For these a far-reaching investigation into the cultural background and oral tradition of specific societies must be substituted. Only in a very few fortunate cases has this stage been attained, and here it is hardly necessary to stress the urgency of attempting this sort of inquiry in all cases where it is not too late. It is only in the third phase of Panofsky's scheme, however, which he calls iconological interpretation, that we reach the "intrinsic meaning or content, constituting the world of "symbolical" values'. It is here that we come into contact with the deeper emotions and feelings of the artist as expressed in his works; and it is here that not only technical and historical knowledge is required of the critic, but also his 'synthetic intuition'. Difficulties arising from attempts at the application of some such method to the study of African sculpture may well bewilder the average Africanist, but it is only through a many-sided analysis of high complexity that we may hope to attain, through a deeper knowledge of this particular class of works, an understanding of the universal values of art.

\section{CONT'RIBUTORS TO THIS NUMBER}

DARYLI Forde. Professor of Anthropology in the University of London; Director of the International African Institute; author of Marriage and the Family among the Yakö, Native Economies in Nigeria, \&c.

Count Vinigi L. Grottanelir. Professor of Ethnology, Pontificium Athenaeum Urbanianum de Propaganda Fide, Rome; Director in the Italian Administration of Antiquities and Fine Arts, and Editor for the section on primitive arts of the Enciclopedia Universale dell' Arte; author of I Mao, Pescatori dell'Oceano Indiano, \&c.

René Lemarchand. Spenta year in the Congo under the auspices of the Ford Foundation at the University of Lovanium; now temporary research assistant at the Institut de Sociologie Solvay, Brussels, where he is completing a doctoral dissertation on the rise and development of Congolese nationalism.

P. M. KaberRY. Reader in Social Anthropology in the University of London; author of Women of the Grassfields, \&c.

E. M. ChILver. Director of the Oxford University Institute of Commonwealth Studies.

W. A. A. WILson. Lecturer in West African Languages at the School of Oriental and African Studies, University of London, 1954-6I; appointed Research Fellow, Institute of African Studies, University of Accra, from October I96I; did field research in Portuguese Guinea 1958-9. 